\title{
Divulgando a Geologia através de roteiros e pontos de interesse geológico: a participação da UERJ no Projeto Caminhos Geológicos
}

Publicizing Geology through Routes and Points of Geological Interest: the participation of UERJ in the Caminhos Geológicos Project

\section{Introdução}

O projeto "Caminhos Geológicos" foi idealizado pelo Departamento de Recursos Minerais do Estado do Rio de Janeiro (DRM/RJ). Em sua concepção ${ }^{1}$, o projeto tinha como objetivos preservar os monumentos geológicos como patrimônios naturais, divulgar o conhecimento geológico, incentivar o desenvolvimento econômico relacionado com a geologia e fortalecer o potencial turístico. Atua principalmente por meio do uso de placas e painéis explicativos em monumentos geológicos (pontos de interesse geológico) em trilhas ou rodovias (Fig. 1 e 2). Quando instaladas em rodovias, os painéis são acompanhados por sinalização oficial do órgão estadual de estradas de rodagem (Fig. 3). Na metodologia inicial também foram propostos roteiros geológicos regionais ${ }^{1}$.

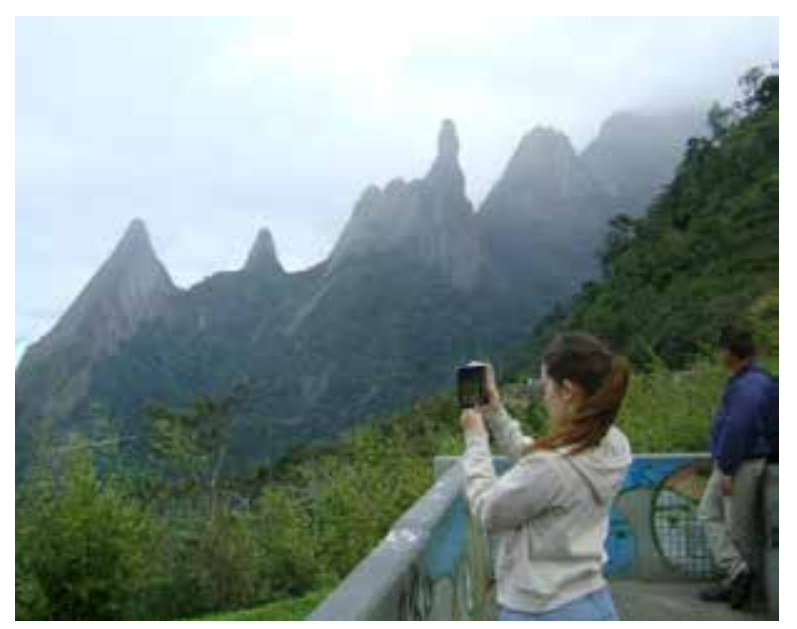

Figura 1: Ponto de Interesse Geológico: perfil montanhoso da Serra dos Órgãos na BR-1 16 visto do Alto do Soberbo, em Teresópolis, RJ, com destaque para o Pico do Dedo de Deus, no centro do perfil
Miguel Tupinambá ${ }^{7}$ e Audrey Alves Monlevade ${ }^{2}$

\section{Resumo}

O projeto Caminhos Geológicos pretende preservar monumentos geológicos e divulgar o conhecimento científico através de painéis explicativos em pontos de interesse geológico. A UERJ colabora com o projeto, realizado em parceria com outras universidades, desde sua criação, com 36 painéis, cerca de $40 \%$ do total já produzido. A intensa participação da UERJ no projeto se deve à prática do curso de Geologia em realizar suas atividades de pesquisa e ensino em território fluminense. A experiência resultou em extensa produção científica sobre a geologia da Faixa Ribeira no Estado do Rio de Janeiro e das bacias da margem continental e culminou com a assinatura de um contrato com o Serviço Geológico do Brasil para mapeamento geológico de todo o estado. O projeto utilizou a experiência de cada autor no ensino e pesquisa da geologia de determinada região fluminense para a divulgação científica. Dessa forma, pretende estender à sociedade o conhecimento geológico adquirido por professores e alunos em treinamentos de campo da graduação, projetos de pós-graduação e pesquisas sobre a Faixa Ribeira e as bacias de Campos e Santos.

Palavras-chave: Divulgação Científica; Caminhos Geológicos; Faixa Ribeira

\section{Área Temática: Meio ambiente}

Linha de Extensão: Patrimônio cultural, histórico e natural 


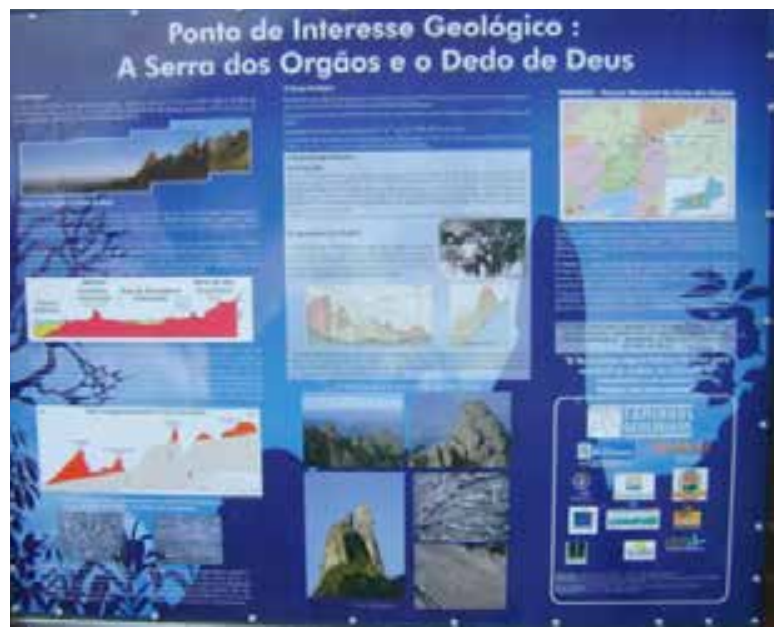

Figura 2: painel explicativo do Ponto de Interesse Geológico instalado junto ao mirante do Alto do Soberbo. A versão em inglês encontra-se na parte traseira da placa

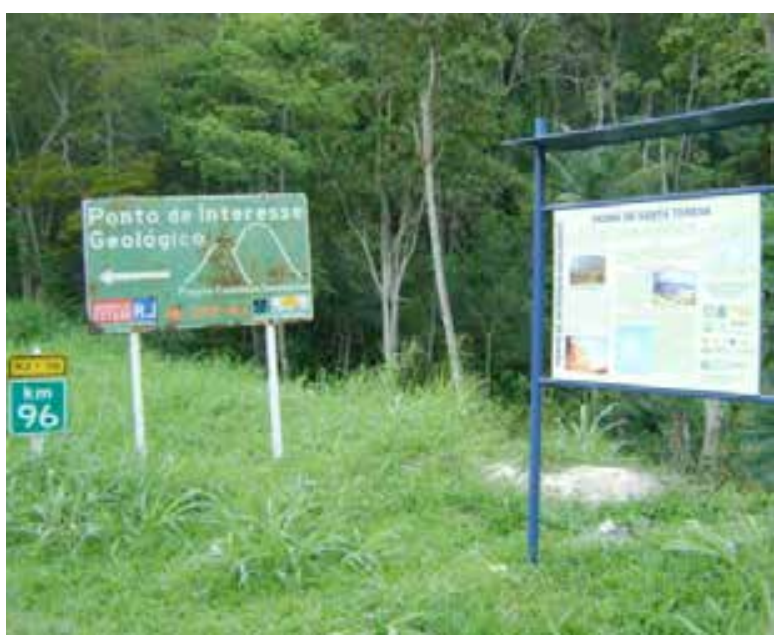

Figura 3: painel explicativo da Pedra da Santa Teresa, no km 96 da rodovia RJ- 116 (Bom Jardim, $\mathrm{RJ})$, ao lado de placa sinalizadora do DER/RJ

O projeto foi concebido contando com dois parceiros fixos, a TURISRIO - Cia. de Turismo do Estado do Rio de Janeiro e o DER - Departamento de Estradas de Rodagem. Também propunha, desde o seu início, parceria científica com as universidades presentes no Estado (UFRJ, UERJ, UFRRJ, UFF), empresas públicas e centros de pesquisas, além do apoio de empresas privadas e prefeituras.

Armação dos Búzios foi o primeiro município a receber placas do projeto Caminhos Geológicos, em agosto de 2001. Em novembro do mesmo ano, sete placas foram instaladas em Santa Maria Madalena, acompanhando o projeto "Cidade da Geo- logia”, do DRM/RJ. Em 2005², 37 painéis haviam sido implantados em 13 municípios fluminenses. Atualmente, o projeto ultrapassa 100 placas em pontos de interesse geológico no interior e na capital fluminense ${ }^{3}$.

A metodologia do projeto também foi adotada em outros estados brasileiros ${ }^{4}$. No Paraná, foi implantado em 2003 o projeto Sítios Geológicos e Paleontológicos do Paraná. Em 2004 e 2005, o Serviço Geológico do Brasil/CPRM inaugurou cinco placas do projeto Caminhos Geológicos da Bahia, mas o projeto não teve continuidade. De 2006 a 2011, o Instituto de Desenvolvimento Econômico e Meio Ambiente do Rio Grande do Norte - IDEM cadastrou 16 monumentos geológicos seu estado5. Em 2007, foi lançado o projeto Monumentos Geológicos da Estrada Real com a instalação de uma placa em Diamantina $(\mathrm{MG})$ sobre a geologia da Serra do Espinhaço. Em 2011, o Serviço Geológico do Brasil/CPRM deu seguimento ao projeto ao apresentar um roteiro geológico na Estrada Real ${ }^{6} \mathrm{com} 32$ pontos de interesses geológico, histórico e turístico. Em São Paulo, o Instituto Geológico/IG executa desde 2009 o projeto Monumentos Geológicos de São Paulo, com a colaboração do Conselho Estadual de Monumentos Geológicos do Estado de São Paulo $(\mathrm{CoMGeo}-\mathrm{SP})^{\text {? }}$.

\section{A Pesquisa Geológica e a Participação da UERJ no Projeto Caminhos Geológicos}

A UERJ é um dos principais parceiros de cooperação técnica do Projeto Caminhos Geológicos do DRM/RJ, dada a participação dos professores e alunos da Faculdade de Geologia na confecção dos painéis. Dos 86 painéis instalados no Estado do Rio de Janeiro 3 , 36 foram confeccionados com produtos da pesquisa geológica da UERJ. No primeiro ano de execução do projeto, em 2001, professores da UERJ foram colaboradores em Armação dos Búzios e Santa Maria Madalena. Desde então, a participação da equipe da UERJ ocorreu em diversos municípios do interior e na capital fluminense (Tab. 1). 
Tabela 1: autoria ou colaboração na redação de Placas do Projeto Caminhos Geológicos (DRM/RJ) de professores e alunos da UERJ

\begin{tabular}{|c|c|c|}
\hline Município & Título & Autor \\
\hline Angra dos Reis & Ilha Grande, a origem de um paraíso & 6 \\
\hline Arraial do Cabo & O Alto de Cabo Frio & 13 \\
\hline Bom Jardim & Pedra de Santa Teresa & 8 \\
\hline Cabo Frio & Limite entre bacias de Campos e Santos & 13,12 \\
\hline Cabo Frio & Parque Boca da Barra, Ilha dos Papagaios & 12,5 \\
\hline Cabo Frio & Forte de São Mateus & 12 \\
\hline Cabo Frio & Ilhas de Cabo Frio & $12,5,13,16$ \\
\hline Casimiro de Abreu & Morro de São João & 13 \\
\hline Iguaba Grande & APA da Serra de Sapiatiba & 12,15 \\
\hline Itaperuna & O Caminho da Pedra Preta & 8,17 \\
\hline Itaperuna & Raposo - a cidade das águas minerais & 12 \\
\hline Macaé & Bacia de Campos & 13 \\
\hline Niterói & Mapa Geológico do Rio de Janeiro & 12 \\
\hline Nova Friburgo & Uma Aventura GeoLÓGICA & 7 \\
\hline Nova Friburgo & Furnas do Catete & 8,4 \\
\hline Nova Friburgo & Quem Riscou a Pedra Riscada? & 7 \\
\hline Nova Friburgo & Quando Ainda Não Existia Floresta & 7 \\
\hline Nova Iguaçu & Feições Misteriosas do Poço do Casarão & 7,1 \\
\hline Nova Iguaçu & Lavas e Brechas Vulcânicas & 7,1 \\
\hline Nova Iguaçu & Geoparque do Vulcão de Nova Iguaçu & 7,3 \\
\hline Nova Iguaçu & Falha do Poço das Cobras & 7,3 \\
\hline Quissamã & Bacia de Campos & 13 \\
\hline Rio das Ostras & Costões Rochosos & 12 \\
\hline Rio de Janeiro & A Odisseia do Pão de Açúcar & 2 \\
\hline Rio de Janeiro & Morro do Corcovado & 2,9 \\
\hline Rio de Janeiro & Geologia do Morro do Corcovado & 2,9 \\
\hline Rio de Janeiro & Pedra do Sal & 3 \\
\hline Sta. Maria Madalena & A Cidade da Geologia & 8,4 \\
\hline Sta. Maria Madalena & Escorrega & 8,4 \\
\hline Sta. Maria Madalena & Pedra Dubois & 8,4 \\
\hline Sta. Maria Madalena & Represa da Tudelândia & 8,4 \\
\hline Sta. Maria Madalena & Serra da Grama & 8 \\
\hline Sto. Antônio Pádua & Rochas Miloníticas & 9,8 \\
\hline São João da Barra & Erosão Marinha em Atafona & 14 \\
\hline Saquarema & 2 Bilhões de Anos de História & 11,4 \\
\hline Teresópolis & Dedo de Deus & 8 \\
\hline
\end{tabular}

Professores: 1 - Ana Maria Netto; 2 - Cláudio de Morisson Valeriano; 3 - Diana Ragatky; 4 - José Renato Nogueira; 5 - Joseffa Varela Guerra (*); 6 - Luiz Guilherme do Eirado Silva; 7 - Mauro César Geraldes; 8 - Miguel Tupinambá; 9 - Mônica Heilbron; 11 - Nely Palermo; 12 - Renata da Silva Schmitt (**); 13 - Webster Mohriak (***). Alunos de graduação em geologia na época da confecção das placas: 14 - Anderson G. de Almeida; 15 - Gilberto da Silva Vaz; 16 - Glauce Menino; 17 - Ingrid Ferreira. $\left({ }^{*}\right)$ professora da Faculdade de Oceanografia da UERJ; (**) endereço atual: Dep. Geologia, Instituto de Geociências da UFRJ; $\left({ }^{* * *}\right)$ professor da UERJ e geólogo da PETROBRÁS. O conteúdo das placas pode ser acessado em http://www.caminhosgeologicos.rj.gov.br. 
A base de conhecimento sobre a geologia fluminense vem sendo construída desde a fundação do curso de Geologia na UERJ em 1977. Neste período, as atividades práticas de campo de diversas disciplinas básicas e aplicadas são executadas parcial ou integralmente em território fluminense. Destacam-se no conjunto os estágios de campo em mapeamento geológico e as aulas de campo de prospecção, geologia econômica e geofísica, que permitiram utilizar o binômio ensino-pesquisa para aprofundar o conhecimento da geologia da Faixa Ribeira e de seus recursos minerais , $^{8,10,11}$. No programa de pós-graduação em Análise de Bacias e Faixas Móveis foram desenvolvidas numerosas dissertações e teses sobre a geologia da Faixa Ribeira em território fluminense e também sobre as bacias marginais de Campos e Santos.

A partir de 2007 a UERJ celebrou contrato com o Serviço Geológico do Brasil/CPRM/PRONAGEO de mapeamento geológico e levantamento de recursos minerais de 13 mapas na escala de 1:100.000, cobrindo cerca de 70\% do território fluminense (Fig. 4). Os mapas e notas explicativas foram publicadas e têm acesso livre em arquivos de diferentes formatos no GEOBANK ${ }^{13}$. Entre
2012 e 2013, a equipe da Faculdade de Geologia da UERJ está elaborando o Mapa Geológico e de Recursos Minerais do Estado do Rio de Janeiro, da série Mapas Geológicos Estaduais do Serviço Geológico do Brasil/CPRM.

O projeto Caminhos Geológicos utilizou a experiência de cada autor no ensino e pesquisa da geologia de determinada região fluminense para a divulgação científica. Na Região Serrana (Nova Friburgo, Bom Jardim, Teresópolis, Santa Maria Madalena) foram aproveitados os trabalhos de Tupinambá ${ }^{10,11}$, Geraldes ${ }^{13}$ e Nogueira ${ }^{14}$. Na região dos lagos fluminense (Cabo Frio, Arraial do Cabo, Iguaba Grande) as placas divulgaram a pesquisa de Schmitt ${ }^{15}$. Na região Noroeste fluminense (Itaperuna, Santo Antonio de Pádua) foram aplicados os dados de Duarte ${ }^{16}$ e Heilbron ${ }^{17}$. Na Região Metropolitana (Rio de Janeiro e Nova Iguaçu) a geologia utilizada é de Valeriano ${ }^{18}$ e Mota ${ }^{19}$. Na região vizinha às bacias de Campos e Santos (Macaé e Quissamã)), foi utilizado o conhecimento geológico offshore de Mohriak ${ }^{20}$.

O contrato de mapeamento geológico e levantamento de recursos Minerais entre a UERJ e o Serviço Geológico do Brasil/CPRM/PRONA-

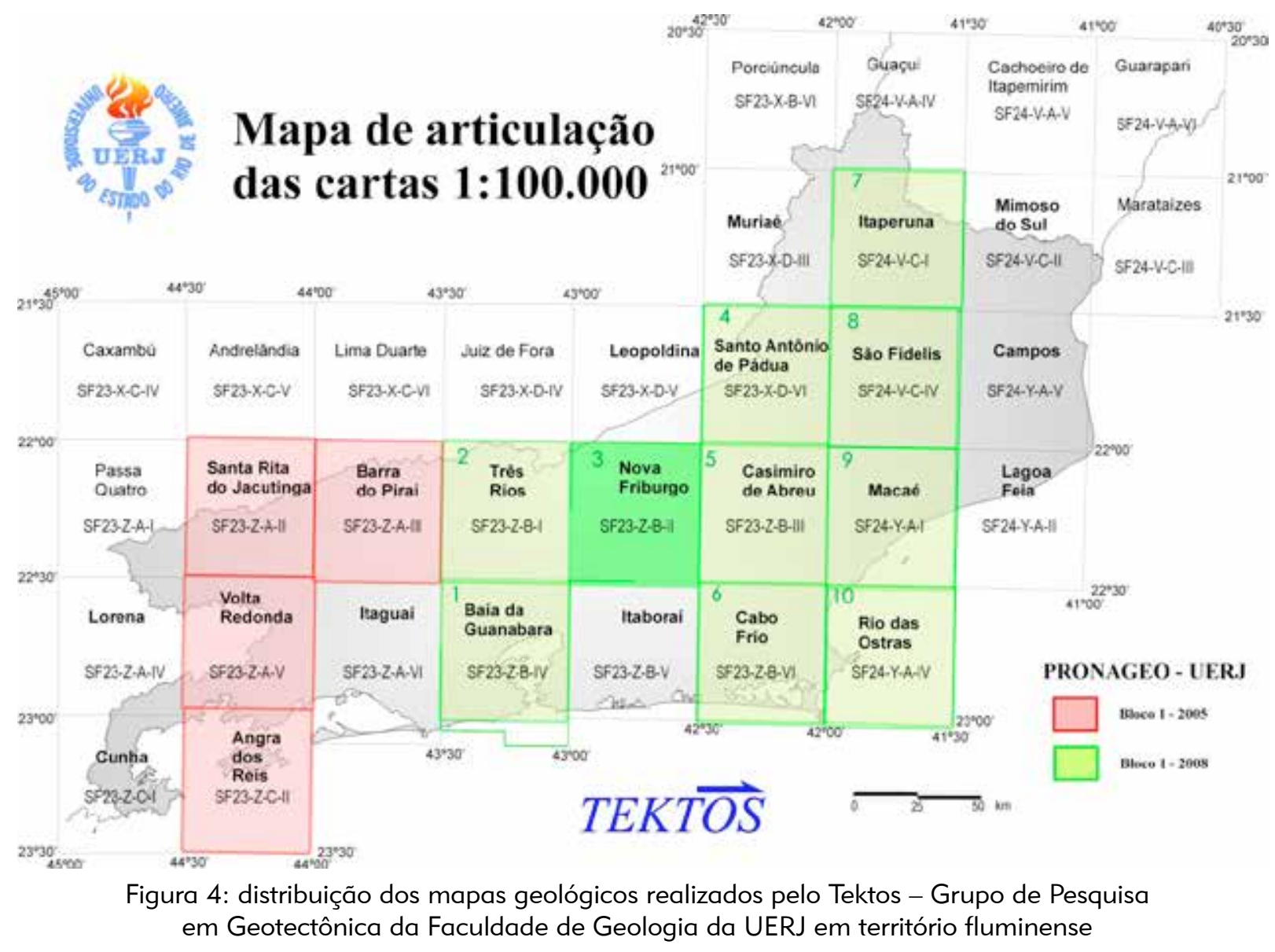


GEO resultou também na publicação, em 2012, de quatro roteiros geoturísticos na região dos $\operatorname{lagos}^{21}$ e na descrição de seis sítios geoturísticos na região metropolitana do Rio de Janeiro, alguns contendo placas em pontos de interesse geológico ${ }^{22}$.

Dessa forma, o projeto integra o trinômio ensino, pesquisa e extensão, estendendo à sociedade o conhecimento geológico adquirido por professores e alunos em treinamentos de campo da graduação, projetos de pós-graduação e pesquisas sobre a Faixa Ribeira e as bacias de Campos e Santos.

\section{Agradecimentos}

O Departamento de Recursos Minerais do Estado do Rio de Janeiro (DRM/RJ) apoia o projeto Caminhos Geológicos na UERJ através de relações institucionais e medidas operacionais para a concretização do projeto. Agradecemos à geóloga Kátia Leite Mansur pela colaboração enquanto coordenava o projeto no DRM e à professora Renata da Silva Schmitt pela implantação e instalação do projeto Caminhos Geológicos na UERJ entre 2002 e 2009. O Departamento de Estradas de Rodagem do Estado do Rio de Janeiro (DER/ RJ) fornece apoio logístico e placas sinalizadoras em rodovias fluminenses. $\mathrm{O}$ projeto tem apoio financeiro do MEC através dos editais PROEXT 2005 e 2011. Agradecemos também à UERJ, representada pelo Departamento de Extensão/SR3, pela concessão de bolsa de extensão a Audrey Monlevade, e ao CNPq por bolsa de produtividade a Miguel Tupinambá. $\mathrm{O}$ projeto tem apoio financeiro do MEC através do PROEXT 2011.

\section{Contribuições dos autores}

Miguel Tupinambá coordena o projeto Caminhos Geológicos na UERJ desde 2009 e é autor ou coautor de 10 placas em pontos de interesse geológico. É de sua autoria a comparação entre produção e divulgação científica dos colaboradores do projeto. Audrey Alves Monlevade é estagiária do contrato MEC/UERJ/PROEXT 2011 e compilou os dados das placas elaboradas pela equipe da UERJ.

\section{Referências}

1. MANSUR, K.; SCHMITT, Renata; ERTHAL, Flávio. Os Caminhos Geológicos do Estado do Rio de Janeiro - um projeto inédito de divulgação científica da geologia no Brasil. In: $7^{\circ} \mathrm{Sim}$ pósio de Geologia do Sudeste, 2001, Rio de Janeiro, Sociedade Brasileira de Geologia, 2001. CD-ROM.

2. MANSUR, Kátia Leite; Guedes, Eliane ; ERTHAL, Flávio, Caminhos Geológicos: um projeto educativo, cultural, turístico, ecológico e científico. In: $9^{\circ}$ Simpósio de Geologia do Sudeste, Niterói. Sociedade Brasileira de Geologia, 2005, CD-ROM.

3. MANSUR, Kátia; NASCIMENTO, Vitor. Projeto Caminhos Geológicos - Inventário geológico do Estado do Rio de Janeiro. Disponível em: <http://www.caminhosgeologicos.rj.gov. br>. Acesso em: 29 jul. 2013, 20:00:00.

4. MANSUR, Kátia Leite. Projetos Educacionais para a Popularização das Geociências e para a Geoconservação. Geologia USP. Publicação Especial, 2009. v. 5, p. 63-74.

5. Instituto Social Iris. Monumentos Geológicos do RN. Disponível em: <http://www.socialiris.org/geologiapotiguar/apresentacao $>$. Acesso em: 26 jul. 2013, 10:43:00.

6. MONTEIRO, Eduardo Araujo. Mapas e Roteiros. In: Excursão Virtual pela Estrada Real no Quadrilátero Ferrífero. Aspectos geológicos, históricos e turísticos. Belo Horizonte, Serviço Geológico do Brasil/CPRM. Disponível em: <http://www. cprm.gov.br/estrada_real/index.html>. Acesso em: 27 jul. 2013, 13:20:00.

7. RIBEIRO, Rogério Rodrigues; GROLA, Diego Amorim. "Projeto Monumentos Geológicos de São Paulo": Uma estratégia de gestão dos Geossítios e Monumentos Geológicos paulistas. In: SBG, Congresso Brasileiro de Geologia, 45, Belém, Anais, CD-ROM. 2010

8. HEILBRON, Monica; SOARES, Antonio Carlos Pedrosa; CAMPOS NETO, Mario; SILVA, Luiz Carlo ; TROUW, Rudolph; JANASI, Valdeci. Província Mantiqueira. In: Virgino Mantesso-Neto; Andrea Bartorelli; Celso Dal Ré Carneiro; Benjamin Bley de Brito Neves. (Org.). Geologia do Continente Sul Americano: Evolução da Obra de Fernando Flávio Marques de Almeida. $1^{a}$ ed.São Paulo: Beca Produções Culturais Ltda., 2004, v. I, p. 203-234.

9. PALERMO, Nely; MANSUR, Katia Leite; GUIMARAES, Paulo; DOURADO, Francisco. Cadastro dos Recursos Minerais da área continental do Estado do Rio de Janeiro. 2. ed. Rio de Janeiro: 2006.

10. TUPINAMBÁ, Miguel; HEILBRON, Monica; DUARTE, Beatriz Paschoal; NOGUEIRA, José Renato; VALLADARES, Claudia; ALMEIDA, Julio; SILVA, Luiz Guilherme Eirado; MEDEIROS, Silvia Regina de; GUIA, Clayton; RAGATKI, Celia Dian ; MENDES, Júlio; LUDKA, Isabel. Geologia da Faixa Ribeira Setentrional: estado da arte e conexões com a Faixa Araçuaí. Geonomos, v. XV, p. 67-79, 2007.

11. TUPINAMBÁ, Miguel; TEIXEIRA, Wilson; HEILBRON, Miguel. Evolução Tectônica e Magmática da Faixa Ribeira entre o Neoproterozoico e o Paleozoico Inferior na Região Serrana do Estado do Rio de Janeiro, Brasil. Anuário do Instituto de Geociências (UFRJ. Impresso), v. 35, p. 140-151, 2012.

12. GONÇALVES, João Henrique. GEOBANK. Belo Horizonte, Serviço Geológico do Brasil/CPRM. Disponível em: $<$ http://www.geobank.sa.cprm.gov.br>. Acesso em: 27 jul. 2013, 13:25:00.

13. GERALDES, Mauro Cesar. Geologia e recursos minerais da folha Casimiro de Abreu SF.23-Z-B-I, estado do Rio de Janeiro escala 1:100.000. 1. Ed. Belo Horizonte: CPRM, 2012. 136p. 
14. NOGUEIRA, José Renato (Org.) Folha São Fidélis, SF.24-V-C-IV: texto e mapa. Programa Geologia do Brasil. 1. ed. Rio de Janeiro: CPRM/UERJ, 2012.

15. SCHMITT, Renata Silva; TROUW, Rudolph; VAN SCHMUS, Wiliam ; PIMENTEL, Marcio Martins .Late amalgamation in the central part of West Gondwana : new geochronological data and the characterization of a Cambrian orogeny in the Ribeira Belt - SE Brazil. Precambrian Research, Amsterdam-Elsevier, v. 133, n.1-2, p. 29-61, 2004.

16. DUARTE, Beatriz Paschoal (Org.). Folha Itaperuna, SF.24-V-C-I: texto e mapa. Programa Geologia do Brasil PGB. 1ed.Rio de Janeiro: CPRM/UERJ, 2012

17. HEILBRON, Monica (Org.). Geologia e Recursos Minerais da Folha Santo Antônio de Pádua-SF.26-X-D-VI. 1 ed.Belo Horizonte: CPRM, 2012

18. VALERIANO, Claudio. (Org.). Geologia e Recursos Minerais da Folha Baía de Guanabara, SF. 23-Z-B-IV, escala 1:100.000.1ed. Belo Horizonte: CPRM, 2012.

19. MOTA, Carlos Eduardo Miranda ; GERALDES, M. C. A classificação de brechas magmáticas e sua implicação na evo- lução do Complexo Alcalino de Nova Iguaçu-RJ. Geociências (São Paulo), v. 25, p. 37-48, 2006.

20. MOHRIAK, Webster. Bacias de Santos, Campos e Espírito Santo. In: Hasui, Yociteru; Carneiro, Celso Dal Ré; Almeida, Fernando Flavio Marques; Bartorelli, Andrea. (Org.). Geologia do Brasil. 1ed.São Paulo: Editora Beca, 2012, v. 1, p. 481-496.

21. MANSUR, Kátia Leite; BARROSO, Sabrina; SCH MITT, Renata da Silva e CERQUEDA, Marcelle Amorim. Geoturismo das Folhas Cabo Frio e Rio das Ostras. In SCH MITT, Renata da Silva (coord.). Geologia e recursos minerais da folha Rio das Ostras SF.24-Y-A-IV e Cabo Frio SF.23-Z-B-VI, Estado do Rio de Janeiro escala 1:100.000. Belo Horizonte: CPRM, 2012.p. 173-190.

22. MANSUR, Kátia Leite; VALERIANO, Cláudio de Morisson. Potencial do Geoturismo na Folha Baia de Guanabara. In VALERIANO, Cláudio de Morisson (coord.). Geologia e recursos minerais da folha Baía de Guanabara SF.23-Z-B-IV, Estado do Rio de Janeiro escala 1:100.000. Belo Horizonte: CPRM, 2012. p. 137-140.

\section{Abstract}

Caminhos Geológicos project aims at preserving geological monuments and disseminating scientific knowledge through explanatory panels at sights of geological interest. It is conducted in partnership with universities and UERJ has cooperated with the project since its implementation with 36 panels - about $40 \%$ of its entire production. The active participation in the project is due to the fact that geological research and teaching practices are located in Rio de Janeiro. The experience resulted not only in extensive scientific production on the geology of the Ribeira Belt in the State of Rio de Janeiro and continental margin basins, but also in earning a contract with Brazil Geological Survey for geological mapping of the entire state. The project used the experience of each author in regional geology of Rio de Janeiro for scientific dissemination. Thus, the project intends to publicize the geological knowledge acquired by professors, undergraduate and graduate students in the Ribeira Belt and the basins of Campos and Santos.

Keywords: Scientific Dissemination; Geological Paths; Ribeira Belt 